\title{
DIVISION VI: INTERSTELLAR MATTER
}

\author{
(MATIERE INTERSTELLAIRE)
}

\author{
PRESIDENT: Bo Reipurth \\ BOARD: Rafael Bachiller, John Dyson (Vice President), José Franco, \\ Thomas Henning, Trung Hua, Sun Kwok, Thomas J. Millar, \\ Guillaume Pineau des Forêts, John Raymond, Yoshiaki Sofue, \\ Grażyna Stasińska, Guillermo Tenorio-Tagle, Silvia Torres-Peimbert \\ \& José Vilchez
}

\section{Commission 34: Interstellar Matter}

\section{Introduction}

Commission 34 covers the Interstellar Matter in its numerous forms, and because of its wide scope it alone forms the Division VI. Subjects under the Division range from all aspects of the tenuous medium between the stars to dense molecular clouds and the processes which form stars to intergalactic matter and clouds. Currently the Division has a membership of around 750. There are at the moment three working groups under Division VI: one on Planetary Nebulae, another on Cosmochemistry, and a third one on Star Formation.

\section{Scientific Highlights}

\subsection{Star Formation}

The subject of star formation has continued its almost explosive growth, and significant new developments have taken place, only a few of which can be mentioned here. Observations by Chandra and XMM-Newton are starting to make an impact, in particular it is noteworthy that X-rays may have been detected from a number of low-mass protostars, offering one more technique to study these deeply embedded objects. The main tool for identifying embedded young stars has up to now been the IRAS catalogue, but with the appearance of the 2MASS sky survey and the MSX galactic plane survey, two new powerful resources have become available. Optical surveys of star forming clouds have now documented that giant Herbig-Haro flows are ubiquitous, and if, as has been suggested, such giant outflows represent dynamical interactions in unstable multiple systems, then young stars may often be formed in small compact groups which subsequently disintegrate. If such dynamical break-up occurs very early in the formation history of a multiple system, the ejected stellar embryos may not have reached sufficient mass to ignite hydrogen burning. This mechanism may be responsible for many of the young brown dwarfs that have recently been detected in star forming regions. Studies of larger scale embedded clusters have improved significantly thanks to the availability of infrared detectors of increasing size, and the infrared luminosity functions of such clusters are becoming better determined. Comparison of infrared excesses in young clusters of different ages suggest that most circumstellar disks become unobservable in the near-infrared after 6-8 millon years. While progress has been substantial in our understanding of low mass star formation, the birth of massive stars is still controversial. The traditional view that massive stars are simply scaled-up versions of low-mass stars is being challenged by the suggestion that massive stars may form through the merging of lower mass protostars in very high density young clusters. 


\subsection{Planetary Nebulae}

Space based observations of planetary nebulae have profoundly affected our understanding of these late stages of stellar evolution. Observations by the Hubble Space Telescope have revealed the presence of crowns, haloes, jets, FLIERs, concentric circular arcs, 2-dimensional rings, and multipolar structures in planetary nebulae. These new discoveries have led to new models for the dynamics and morphologies of planetary nebulae. Observations by the Infrared Space Observatory have revealed complex organic and inorganic compounds in planetary nebulae. These results show that molecular synthesis in the circumstellar environment can occur over time scales of hundreds to thousands of years, and such synthesis may play a major role in the chemical enrichment of the interstellar medium. Planetary nebulae are now extensively used as standard candles to determine the Hubble constant, to trace the distribution of dark matter in galaxies, and to determine the mass of intracluster matter. Finally, we note the discovery of the most oxygen poor planetary nebula known so far, with an oxygen abundance around $1 / 500$ the solar value, similar to those of the most oxygen poor stars.

\subsection{HII Regions and other Nebulae}

Chandra observations have been applied to the study of the interstellar medium related to SNRs, PN, HII regions and HII galaxies, revealing the distribution of hot gas and, in the case of giant HII regions and HII galaxies, the possible footprints of a metal enriched outflow. Much attention has been devoted to the temperature fluctuation conundrum and the recombination versus collisional excitation discrepancy, which continue to be the main problems in nebular astronomy, but there is still no clear and generally accepted solution. ISO results have considerably enriched the observational data base on nebulae, and emission lines in the infrared have provided new diagnostics of abundances in HII regions and planetary nebulae.

\subsection{ISM in Galaxies}

Detailed studies of the molecular component of the ISM in other galaxies have long been hampered by poor resolution. Major progress is due to interferometric observations in the CO line under the BIMA Survey of Nearby Galaxies which have provided maps with 4- ${ }^{\prime \prime}$ resolution, allowing comparison with optical and infrared images emphasizing recent star formation. Also, the Virgo high-resolution CO-line survey with the Nobeyama mm-wave Array at $2-3^{\prime \prime}$ resolution was completed, finding high-velocity nuclear rotations indicative of massive cores.

\subsection{ALMA}

The bilateral (European-North American) project to construct the Atacama Large Millimeter Array has taken major steps during the period $2000-2002$, and now moves into its second phase, construction. With its 64 12-meter high-precision antennas employing baselines of up to $12 \mathrm{~km}$, ALMA will be a millimeter/submillimeter match to the opti$\mathrm{cal} /$ infrared 8-meter to 10 -meter class telescopes that have emerged in recent years. The receivers are chosen to cover the atmospheric windows between 0.3 and 10 millimeters, taking full advantage of the superb location that has been identified at 5000 meter altitude near Cerro Chajnantor in northern Chile. When completed, ALMA will offer the astronomical community unprecedented opportunities to study, among others, star forming cloud cores, circumstellar disks and planet formation, envelopes of evolved stars, shock structures, and extragalactic molecular clouds at low and high redshift. It is therefore poised to become a prime tool for the science pursued in Division VI. 


\section{Working Groups}

Planetary Nebula Working Group: Chair: Sun Kwok (Canada);

Agnes Acker (France), Michael J. Barlow (UK), George Jacoby (USA), Jim Kaler (USA), Walter Maciel (Brasil), Dipankar Mallik (India), Mario Perinotto (Italy), Stuart Pottasch (Netherlands), Luis Rodríguez (Mexico), Detlef Schönberner (Germany), Yervant Terzian (USA), Romuald Tylenda (Poland), and Peter Wood (Australia).

At the last working group meeting a formal mechanism for the rotation of the membership of the WG organizing committee was adopted. It was resolved that the organizing committee will consist of 15 members, with 5 members rotating off and 5 new members elected every 5 years. At the end of each 5 -year term, a new chairman will be elected. The chairman will be responsible for organizing the next IAU Symposium on planetary nebulae and will serve as chairman or co-chairman of the SOC. The website of the Planetary Nebula Working Group is at: http://www.iras.ucalgary.ca/pnwg/pnwg.html

Astrochemistry Working Group: Chair: Ewine van Dishoeck (The Netherlands); L. Allamandola (USA), J.H. Black (Sweden), G.A. Blake (USA), P. Caselli (Italy), P. Ehrenfreund (The Netherlands), G. Garay (Chile), M. Guelin (France), C. Henkel (Germany), U. G. Jorgensen (Denmark), J.P. Maier (Switzerland), K.M. Menten (Germany), T.J. Millar (UK), Y.C. Minh (S. Korea), M. Ohishi (Japan), A. Raga (Mexico), J. Rawlings (UK), B. Rowe (France), J. Yang (China).

The main activity of the IAU Astrochemistry working group in the period July 11999 to June 302002 was the organization of IAU Symposium 197 on "Astrochemistry: From Molecular Clouds to Planetary Systems". This symposium took place August 23-27 1999 in Sogwipo, South Korea, and was attended by 260 participants from at least 25 countries and nationalities. The proceedings, edited by Y.C. Minh and E.F. van Dishoeck and published in 2000, contain an excellent overview of the field. The Astrochemistry working group was also responsible for stimulating and supporting numerous other smaller meetings in this research area over the last three years, and for maintaining a Web site with link to molecular data bases: http://www.strw.leidenuniv.nl/ iau34/.

Star Formation Working Group: Chair: Francesco Palla (Italy);

B. Reipurth (Secretary, USA), R. Bachiller (Spain), M. Burton (Australia), L. Cram (Australia), Y. Fukui (Japan), G. Garay (Chile), T. Henning (Germany), C.J. Lada (USA), M.T. Lago (Portugal), S. Lizano (Mexico), J. Palouš (Czechia), A. Sargent (USA), S. Strom (USA).

The main activity of the Star Formation Working Group in the period 2000-2002 has been stimulation and support of meetings in this area, including IAU Symposium 211 on "Brown Dwarfs" held in Hawaii in May 2002, and IAU Symposium 221 on "Star Formation at High Angular Resolution" that will take place during the IAU General Assembly in 2003 (Sydney, Australia). Support was also given in the preparation of two Joint Discussions on "Formation of Cometary Material" (JD 14) and "The Astrochemistry of External Galaxies" (JD 21) that will be held at the IAU-GA of 2003. The Star Formation Working Group currently has its web site at http://www.arcetri.astro.it/sfwg/

\section{Major Conferences}

A great number of symposia and workshops related to the interstellar medium were held during the past three years, and it is impossible to make a complete compilation. Selected meetings are listed below.

In the area of star formation the following conferences were held: ESO Workshop on The Origin of Stars and Planets: The VLT View, on 24-27 April 2001, held in Garching, Germany; The Earliest Stages of Massive Star Birth, on 6-8 August 2001, held in Boulder, Colorado, USA; Galactic Star Formation across the Stellar Mass Spectrum, on 11-15 March, 2002, held in La Serena, Chile; Debris Disks and the Formation of Planets: A Symposium 
in Memory of Fred Gillett, on 11-13 April 2002, held in Tucson, Arizona, USA; Star Formation Workshop 2002: Magnetohydrodynamics, Radiation Diagnostics, and Chemistry of Star Formation, on 12-17 June 2002, held in Taiwan; Chemistry as a Diagnostic of Star Formation, on 21-23 August 2002, held in Waterloo, Canada.

Several meetings were devoted to planetary nebulae and late stellar evolutionary phases: Asymmetric planetary nebulae II: from origins to microstructures, on 3-6 August, 1999, held in Cambridge, USA; Post-AGB objects as a phase of stellar evolution, on 5-7 July 2000, held in Torun, Poland; IAU Symposium 209 on Planetary Nebulae, their evolution and role in the Universe, on 19-23 November 2001, held in Canberra, Australia.

Other meetings on various aspects of the interstellar medium include: Astrophysical Plasmas: Codes, Models $\mathcal{B}$ Observations, on 25-29 October 1999, held in Mexico City, Mexico; Emission Lines from Jet Flows, on 13-17 November 2000, held in Isla Mujeres, Mexico; Ionized Gaseous Nebulae, on 21-24 November 2000, held in Mexico City, Mexico; Winds, Bubbles and Explosions: a conference to honour John Dyson, on 9-13 September 2002, held in Pátzcuaro, México.

The interstellar medium in galaxies was dealt with in the following conferences: The series of 3 Euro-Conferences on The Evolution of Galaxies I; II; III, the first was held in Granada 2000, the second on La Reunion Island 2001, and the third in Kiel 2002; Mapping the Hidden Universe on 23-29 February 2000, held in Guanajuato, Mexico; Vatican Conference on Galaxy Disks and Disk Galaxies, on 12-16 June 2000, held in Rome, Italy; Ringberg Work Shop on Virgo Cluster of Galaxies, on 14-17 April 2001, held at Ringberg Castle, Germany; Disks of Galaxies: Kinematics, Dynamics and Perturbations, on 5-9 November 2001, held in Puebla, Mexico; Galaxies: The Third Dimension, on 3-7 December 2001, held in Cozumel, Mexico; CNO in the Universe, 10-14 September 2002, held in SaintLuc, Switzerland.

\section{Major Publications}

A new catalogue, the Edinburgh-AngloAustralianObservatory-Strasbourg Catalogue of New and Possible Planetary Nebulae, reports over 900 new planetary nebulae discovered from the AAO/UKST H-alpha survey of the Southern Galactic Plane. A second release of the catalogue, expected in 2003, will double the total number of galactic planetary nebulae discovered from all sources in the last 75 years.

\section{Further Sources}

"The Star Formation Newsletter" is a monthly electronic publication which provides abstracts of recently accepted papers in the field of star formation, molecular clouds and the interstellar medium. All issues can be found at http://www.eso.org/gen-fac/pubs/starform/

"The Herbig-Bell Catalogue" lists coordinates, information and references to the literature on 735 pre-main sequence stars. It can be found at http://www-astro.phast.umass.edu/catalogs/HBC/HBC.html

"A General Catalogue of Herbig-Haro Objects" is a complete list of all known HerbigHaro objects, with coordinates, extensive notes and a full list of references to the literature for each object. The catalogue, which is now in its 2 . edition, will be updated every few years. It can be downloaded from http://casa.colorado.edu/hhcat

Other useful links to star formation related research can be found at the web site of the Center for Star and Planet Formation at the Institute for Astronomy of the University of Hawaii. It can be found at http://www2.ifa.hawaii.edu/CSPF/. Among other things, this web site gives links to numerous catalogues, books, dissertations and major review articles. It also lists all upcoming meetings relevant to star and planet formation.

Bo Reipurth

President of the Division 